\title{
Wreath product action on generalized Boolean algebras
}

\author{
Ashish Mishra and Murali K. Srinivasan \\ Department of Mathematics \\ Indian Institute of Technology \\ Mumbai 400076, India \\ ashishm@math.iitb.ac.in \\ murali.k.srinivasan@gmail.com
}

Submitted: Nov 15, 2014; Accepted: May 23, 2015; Published: Jun 3, 2015

Mathematics Subject Classifications: 05E10, 05E25

\begin{abstract}
Let $G$ be a finite group acting on the finite set $X$ such that the corresponding (complex) permutation representation is multiplicity free. There is a natural rank and order preserving action of the wreath product $G \sim S_{n}$ on the generalized Boolean algebra $B_{X}(n)$. We explicitly block diagonalize the commutant of this action.
\end{abstract}

\section{Introduction}

This paper is inspired by the following two results:

(i) Explicit diagonalization of the "Bose-Mesner algebra" (= commutant of a certain wreath product action) of the generalized Johnson scheme, by Ceccherini-Silberstein, Scarabotti, and Tolli [2].

(ii) Explicit block diagonalization of the commutant of the wreath product action on the nonbinary analog of the Boolean algebra, due to Dunkl [5] and Gijswijt, Schrijver, and Tanaka [6].

A natural question suggested by these results is to explicitly block diagonalize the commutant of the wreath product action on the generalized Boolean algebra. To do that is one of the aims of this paper. Our second aim is to recast the results of [2] on the generalized Johnson scheme, presented there in the language of harmonic analysis (Gelfand pairs, induced representations, spherical functions etc.), in purely combinatorial terms. This is achieved by means of the concepts of semisymmetric Jordan basis and upper Boolean decomposition. These notions are implicit in $[\mathbf{2}, \mathbf{6}]$ and were stated explicitly in [10]. They allow a simple reduction to the case of symmetric group action on Boolean algebras, 
given in $[5,6]$. In the rest of this introduction we define our objects of study and state our result.

Let $\operatorname{Mat}(n \times n)$ denote the algebra of complex $n \times n$ matrices and let $\mathcal{A} \subseteq \operatorname{Mat}(n \times n)$ denote a $*$-subalgebra. Then the noncommutative analog of the spectral theorem asserts that there exists a block diagonalization of $\mathcal{A}$, i.e., there exists a $\{1, \ldots, n\} \times S$ unitary matrix $N$, for some index set $S$ of cardinality $n$, and positive integers $p_{0}, q_{0}, \ldots, p_{m}, q_{m}$ such that $N^{*} \mathcal{A} N$ is equal to the set of all $S \times S$ block-diagonal matrices

$$
\left(\begin{array}{cccc}
C_{0} & 0 & \ldots & 0 \\
0 & C_{1} & \ldots & 0 \\
\vdots & \vdots & \ddots & \vdots \\
0 & 0 & \ldots & C_{m}
\end{array}\right)
$$

where each $C_{k}$ is a block-diagonal matrix with $q_{k}$ repeated, identical blocks of order $p_{k}$

$$
C_{k}=\left(\begin{array}{cccc}
B_{k} & 0 & \ldots & 0 \\
0 & B_{k} & \ldots & 0 \\
\vdots & \vdots & \ddots & \vdots \\
0 & 0 & \ldots & B_{k}
\end{array}\right)
$$

Thus $p_{0}^{2}+\cdots+p_{m}^{2}=\operatorname{dim} \mathcal{A}$ and $p_{0} q_{0}+\cdots+p_{m} q_{m}=n$. The numbers $p_{0}, q_{0}, \ldots, p_{m}, q_{m}$ and $m$ are uniquely determined (upto permutation of the indices) by $\mathcal{A}$.

By dropping duplicate blocks we get a $*$-isomorphism

$$
\Phi: \mathcal{A} \cong \bigoplus_{k=0}^{m} \operatorname{Mat}\left(p_{k} \times p_{k}\right) .
$$

In an explicit block diagonalization we need to know this isomorphism explicitly, i.e., we need to know the entries in the image $\Phi(M)$, for $M$ varying over a suitable basis of $\mathcal{A}$. When $\mathcal{A}$ is commutative we have $p_{k}=1$ for all $k$ and we speak of explicit diagonalization.

We now define the $*$-algebras to be considered in this paper. Let $B(n)$ denote the set of all subsets of $[n]=\{1,2, \ldots, n\}$. For a finite set $S$, let $V(S)$ denote the complex vector space with $S$ as basis.

Let $G$ be a finite group acting on the finite set $X$. Assume that the corresponding permutation representation on $V(X)$ is multiplicity free. This implies, in particular, that the action is transitive.

Let $L_{0}$ be a symbol not in $X$ and let $Y$ denote the alphabet $Y=\left\{L_{0}\right\} \cup X$. We call the elements of $X$ the nonzero letters in $Y$. Define $B_{X}(n)=\left\{\left(a_{1}, \ldots, a_{n}\right): a_{i} \in Y\right.$ for all $\left.i\right\}$, the set of all $n$-tuples of elements of $Y$ (we use $L_{0}$ instead of 0 for the zero letter for later convenience. We do not want to confuse the letter 0 with the vector 0$)$. Given $a=\left(a_{1}, \ldots, a_{n}\right) \in B_{X}(n)$, define the support of $a$ by $S(a)=\left\{i \in[n]: a_{i} \neq L_{0}\right\}$. For $0 \leqslant i \leqslant n, B_{X}(n)_{i}$ denotes the set of all elements $a \in B_{X}(n)$ with $|S(a)|=i$. We have

$$
\left|B_{X}(n)\right|=(|X|+1)^{n}, \quad\left|B_{X}(n)_{i}\right|=\left(\begin{array}{c}
n \\
i
\end{array}\right)|X|^{i} .
$$


(We take the binomial coefficient $\left(\begin{array}{l}n \\ k\end{array}\right)$ to be 0 if $n<0$ or $k<0$ ).

Let $S_{n}$ denote the symmetric group on $n$ letters. There is a natural action of the wreath product $G \sim S_{n}$ (see $\left.[3,7]\right)$ on $B_{X}(n)$ and $B_{X}(n)_{i}$ : permute the $n$ coordinates followed by independently acting on the nonzero letters by elements of $G$. In detail, given $\left(g_{1}, g_{2}, \ldots, g_{n}, \pi\right) \in G \sim S_{n}$ (where $\pi \in S_{n}$ and $g_{i} \in G$ for all $i$ ) and $a=\left(a_{1}, \ldots, a_{n}\right) \in$ $B_{X}(n)$, we have $\left(g_{1}, \ldots, g_{n}, \pi\right)\left(a_{1}, \ldots, a_{n}\right)=\left(b_{1}, \ldots, b_{n}\right)$, where $b_{i}=g_{i} a_{\pi^{-1}(i)}$, if $a_{\pi^{-1}(i)}$ is a nonzero letter and $b_{i}=L_{0}$, if $a_{\pi^{-1}(i)}=L_{0}$.

We give $V(Y)$ and $V\left(B_{X}(n)\right)$ the standard inner products, i.e., we declare $Y$ and $B_{X}(n)$ to be orthonormal bases.

We represent elements of $\operatorname{End}\left(V\left(B_{X}(n)\right)\right.$ ) (in the standard basis $\left.B_{X}(n)\right)$ as $B_{X}(n) \times$ $B_{X}(n)$ matrices (we think of $V\left(B_{X}(n)\right)$ as column vectors with coordinates indexed by $\left.B_{X}(n)\right)$. For $a, b \in B_{X}(n)$, the entry in row $a$, column $b$ of a matrix $M$ will be denoted $M(a, b)$. The matrix corresponding to $f \in \operatorname{End}\left(V\left(B_{X}(n)\right)\right)$ will be denoted $M_{f}$. We use similar notations for $B_{X}(n)_{i} \times B_{X}(n)_{i}$ matrices corresponding to elements of $\operatorname{End}\left(V\left(B_{X}(n)_{i}\right)\right)$.

Set

$$
\begin{aligned}
\mathcal{A}_{X}(n) & =\left\{M_{f}: f \in \operatorname{End}_{G \sim S_{n}}\left(V\left(B_{X}(n)\right)\right)\right\} \\
\mathcal{B}_{X}(n, i) & =\left\{M_{f}: f \in \operatorname{End}_{G \sim S_{n}}\left(V\left(B_{X}(n)_{i}\right)\right)\right\} .
\end{aligned}
$$

Thus $\mathcal{A}_{X}(n)$ and $\mathcal{B}_{X}(n, i)$ are $*$-algebras of matrices.

In the paper [2] it is shown that the action of $G \sim S_{n}$ on $V\left(B_{X}(n)_{i}\right)$ is multiplicity free and the commutant $\mathcal{B}_{X}(n, i)$ of this action is explicitly diagonalized. This generalizes the case of the Johnson scheme [4] (corresponding to $|X|=1$ ) and the nonbinary Johnson scheme [13] (corresponding to $G=$ group of all permutations of $X$ ). For other examples of this framework see $[\mathbf{2}, \mathbf{3}]$. We consider the related problem of explicitly block diagonalizing the commutant $\mathcal{A}_{X}(n)$ of the action of $G \sim S_{n}$ on $V\left(B_{X}(n)\right)$. In the process we also present an alternative combinatorial approach to the generalized Johnson scheme of [2].

Let $f: V\left(B_{X}(n)\right) \rightarrow V\left(B_{X}(n)\right)$ be linear. Then $f$ is $G \sim S_{n}$-linear if and only if

$$
M_{f}(a, b)=M_{f}(\tau(a), \tau(b)) \text {, for all } a, b \in B_{X}(n), \tau \in G \sim S_{n},
$$

i.e., $M_{f}$ is constant on the orbits of the action of $G \sim S_{n}$ on $B_{X}(n) \times B_{X}(n)$. We now determine these orbits.

Denote the orbits of $G$ on $X \times X$ by

$$
Z_{0}, Z_{1}, \ldots, Z_{m}
$$

where $Z_{0}=\{(x, x): x \in X\}$. Note that $m=0$ iff $|X|=1$. Unless otherwise stated we shall assume $m \geqslant 1$ (or, equivalently, $|X| \geqslant 2$ ). The theory to be presented in this paper solves the $|X| \geqslant 2$ case in terms of the $|X|=1$ case (which is summarized in Section 2 below).

Let

$$
C(n, m)=\left\{\left(l_{1}, \ldots, l_{m}\right) \in \mathbb{N}^{m}: l_{1}+\cdots+l_{m}=n\right\},
$$


where $\mathbb{N}=\{0,1,2, \ldots\}$, denote the set of all weak compositions of $n$ with $m$ parts. We have $|C(n, m)|=\left(\begin{array}{c}n+m-1 \\ n\end{array}\right)=\left(\begin{array}{c}n+m-1 \\ m-1\end{array}\right)$.

Given $(a, b) \in B_{X}(n) \times B_{X}(n)$, where $a=\left(a_{1}, \ldots, a_{n}\right)$ and $b=\left(b_{1}, \ldots, b_{n}\right)$, define

$$
\mathcal{C}(a, b)=\left(l_{0}, \ldots, l_{m}\right) \in C(|S(a) \cap S(b)|, m+1)
$$

by

$$
l_{j}=\left|\left\{i \in S(a) \cap S(b):\left(a_{i}, b_{i}\right) \in Z_{j}\right\}\right|, j=0, \ldots, m .
$$

It is now easily seen that $(a, b),(c, d) \in B_{X}(n) \times B_{X}(n)$ are in the same $G \sim S_{n}$-orbit if and only if

$$
|S(a)|=|S(c)|,|S(b)|=|S(d)|,|S(a) \cap S(b)|=|S(c) \cap S(d)|, \mathcal{C}(a, b)=\mathcal{C}(c, d) .
$$

For $0 \leqslant i, j, t \leqslant n$ and $l=\left(l_{0}, \ldots, l_{m}\right) \in \mathbb{N}^{m+1}$ let $M_{i, j}^{t, l}$ be the $B_{X}(n) \times B_{X}(n)$ matrix given by

$$
M_{i, j}^{t, l}(a, b)= \begin{cases}1 & \text { if }|S(a)|=i,|S(b)|=j,|S(a) \cap S(b)|=t, \mathcal{C}(a, b)=l, \\ 0 & \text { otherwise. }\end{cases}
$$

Define

$$
\mathcal{I}_{X}(n)=\{(i, j, t, l): i, j, t \in \mathbb{N}, t \leqslant i, t \leqslant j, i+j-t \leqslant n, l \in C(t, m+1)\} .
$$
have

It follows from (3) and (4) that $\left\{M_{i, j}^{t, l}:(i, j, t, l) \in \mathcal{I}_{X}(n)\right\}$ is a basis of $\mathcal{A}_{X}(n)$. We

$$
\left|\mathcal{I}_{X}(n)\right|=\operatorname{dim} \mathcal{A}_{X}(n)=\left(\begin{array}{c}
n+m+3 \\
m+3
\end{array}\right),
$$

since $(i, j, t, l) \in \mathcal{I}_{X}(n)$ if and only if $(i-t)+(j-t)+l_{0}+\cdots+l_{m} \leqslant n$ and all $m+3$ terms are nonnegative.

For $0 \leqslant i \leqslant n$ define

$$
\mathcal{I}_{X}(n, i)=\{(t, l): t \in \mathbb{N}, t \leqslant i, 2 i-t \leqslant n, l \in C(t, m+1)\} .
$$

We have that $\left\{M_{i, i}^{t, l}:(t, l) \in \mathcal{I}_{X}(n, i)\right\}$ is a basis of $\mathcal{B}_{X}(n, i)$ (here we think of $M_{i, i}^{t, l}$ as $B_{X}(n)_{i} \times B_{X}(n)_{i}$ matrices). We have

$$
\operatorname{dim} \mathcal{B}_{X}(n, i)= \begin{cases}\sum_{t=0}^{i}|C(t, m+1)|=\left(\begin{array}{c}
m+i+1 \\
m+1
\end{array}\right), & \text { if } i \leqslant n / 2 \\
\sum_{t=2 i-n}^{i}|C(t, m+1)|=\left(\begin{array}{c}
m+i+1 \\
m+1
\end{array}\right)-\left(\begin{array}{c}
m+2 i-n \\
m+1
\end{array}\right), & \text { if } i>n / 2\end{cases}
$$

where we have used the identity $\sum_{t=0}^{i}\left(\begin{array}{c}m+t \\ t\end{array}\right)=\left(\begin{array}{c}m+i+1 \\ m+1\end{array}\right)$ (Exercise 3(a) in Chapter 1 of $[12])$.

Note that if $m=0$ or 1 then, for $x, y \in X,(x, y)$ and $(y, x)$ are in the same $G$-orbit of $X \times X$. Thus $M_{i, i}^{t, l}$ is symmetric and it follows that $\mathcal{B}_{X}(n, i)$ is commutative, since a subalgebra of Mat $(n \times n)$ having a basis consisting of symmetric matrices is commutative. 
Hence $V\left(B_{X}(n)_{i}\right)$ is a multiplicity free $G \sim S_{n}$-module. This argument does not apply when $m \geqslant 2$.

We now describe our explicit block diagonalization of $\mathcal{A}_{X}(n)$. We need to make a number of definitions.

Our poset terminology follows [12]. Let $P$ be a finite graded poset with rank function $r: P \rightarrow \mathbb{N}$. The rank of $P$ is $r(P)=\max \{r(x): x \in P\}$ and, for $i=0,1, \ldots, r(P), P_{i}$ denotes the set of elements of $P$ of rank $i$. For a subset $S \subseteq P$, we set $\operatorname{rankset}(S)=$ $\{r(x): x \in S\}$.

Let $P$ be a graded poset with $n=r(P)$. Then we have $V(P)=V\left(P_{0}\right) \oplus$ $V\left(P_{1}\right) \oplus \cdots \oplus V\left(P_{n}\right)$ (vector space direct sum). An element $v \in V(P)$ is homogeneous if $v \in V\left(P_{i}\right)$ for some $i$, and if $v \neq 0$, we extend the notion of rank to nonzero homogeneous elements by writing $r(v)=i$. Given an element $v \in V(P)$, write $v=v_{0}+\cdots+v_{n}, \quad v_{i} \in V\left(P_{i}\right), 0 \leqslant i \leqslant n$. We refer to the $v_{i}$ as the homogeneous components of $v$. A subspace $W \subseteq V(P)$ is homogeneous if it contains the homogeneous components of each of its elements. For a homogeneous subspace $W \subseteq V(P)$ we set $\operatorname{rankset}(W)=\{r(v): v$ is a nonzero homogeneous element of $W\}$.

The up operator $U: V(P) \rightarrow V(P)$ is defined, for $x \in P$, by $U(x)=\sum_{y} y$, where the sum is over all $y$ covering $x$. Similarly, the down operator $D: V(P) \rightarrow V(P)$ is defined, for $x \in P$, by $D(x)=\sum_{y} y$, where the sum is over all $y$ covered by $x$.

Let $\langle$,$\rangle denote the standard inner product on V(P)$, i.e., $\langle x, y\rangle=\delta(x, y)$ (Kronecker delta), for $x, y \in P$. The length $\sqrt{\langle v, v\rangle}$ of $v \in V(P)$ is denoted $\|v\|$.

Let $P$ be a graded poset of rank $n$. A graded Jordan chain in $V(P)$ is a sequence

$$
c=\left(v_{1}, \ldots, v_{h}\right)
$$

of nonzero homogeneous elements of $V(P)$ such that $U\left(v_{i-1}\right)=v_{i}$, for $i=2, \ldots, h$, and $U\left(v_{h}\right)=0$ (note that the elements of this sequence are linearly independent, being nonzero and of different ranks). We say that $c$ starts at rank $r\left(v_{1}\right)$ and ends at rank $r\left(v_{h}\right)$. A graded Jordan basis of $V(P)$ is a basis of $V(P)$ consisting of a disjoint union of graded Jordan chains in $V(P)$.

The graded Jordan chain (7) is said to be a symmetric Jordan chain (SJC) if the sum of the starting and ending ranks of $c$ equals $n$, i.e., $r\left(v_{1}\right)+r\left(v_{h}\right)=n$ if $h \geqslant 2$, or $2 r\left(v_{1}\right)=n$ if $h=1$. A symmetric Jordan basis (SJB) of $V(P)$ is a basis of $V(P)$ consisting of a disjoint union of symmetric Jordan chains in $V(P)$.

The graded Jordan chain (7) is said to be a semisymmetric Jordan chain (SSJC) if the sum of the starting and ending ranks of $c$ is $\geqslant n$. A semisymmetric Jordan basis (SSJB) of $V(P)$ is a basis of $V(P)$ consisting of a disjoint union of semisymmetric Jordan chains in $V(P)$.

For $0 \leqslant k \leqslant n$ we set $k^{-}=\max (0,2 k-n)$ (note that $k^{-}$depends on both $k$ and $n$. The $n$ will always be clear from the context). Note that $0 \leqslant k^{-} \leqslant k$ and $k \leqslant n+k^{-}-k$. For a SSJC $c$ in $V(P)$, starting at rank $i$ and ending at rank $j$, we define the offset of $c$ to be $i+j-n$. It is easy to see that if an SSJC of offset $s$ starts at rank $k$ then the chain ends at rank $n+s-k$ and we have $k^{-} \leqslant s \leqslant k$. Also note that the conditions $0 \leqslant k \leqslant n, k^{-} \leqslant s \leqslant k$ are easily seen to be equivalent to $0 \leqslant s \leqslant k \leqslant\left\lfloor\frac{n+s}{2}\right\rfloor \leqslant n$. 
We use the following terminology in connection with the notion of block diagonal form: let $A$ be a finite set and let $N$ be a $A \times A$ matrix. Given a partition $A=A_{1} \cup \cdots \cup A_{k}$ of $A$ into pairwise disjoint nonempty subsets $A_{1}, \ldots, A_{k}$ we say that $N$ is in block diagonal form with a block corresponding to each $A_{i}$ if $A(x, y)=0$ whenever $(x, y) \notin\left(A_{1} \times A_{1}\right) \cup$ $\cdots \cup\left(A_{k} \times A_{k}\right)$.

Suppose we have an orthogonal graded Jordan basis $O$ of $V(P)$. Normalize the vectors in $O$ to get an orthonormal basis $O^{\prime}$. Let $\left(v_{1}, \ldots, v_{h}\right)$ be a graded Jordan chain in $O$. Put $v_{u}^{\prime}=\frac{v_{u}}{\left\|v_{u}\right\|}$ and $\alpha_{u}=\frac{\left\|v_{u+1}\right\|}{\left\|v_{u}\right\|}, 1 \leqslant u \leqslant h$ (we take $v_{0}^{\prime}=v_{h+1}^{\prime}=0$ ). We have, for $1 \leqslant u \leqslant h$,

$$
U\left(v_{u}^{\prime}\right)=\frac{U\left(v_{u}\right)}{\left\|v_{u}\right\|}=\frac{v_{u+1}}{\left\|v_{u}\right\|}=\alpha_{u} v_{u+1}^{\prime} .
$$

Thus the matrix of $U$ with respect to $O^{\prime}$ is in block diagonal form, with a block corresponding to each (normalized) graded Jordan chain in $O$, and with the block corresponding to $\left(v_{1}^{\prime}, \ldots, v_{h}^{\prime}\right)$ above being a lower triangular matrix with subdiagonal $\left(\alpha_{1}, \ldots, \alpha_{h-1}\right)$ and 0 's elsewhere.

Now note that the matrices, in the standard basis $P$, of $U$ and $D$ are real and transposes of each other. Since $O^{\prime}$ is orthonormal with respect to the standard inner product, it follows that the matrices of $U$ and $D$, in the basis $O^{\prime}$, must be adjoints of each other. Thus the matrix of $D$ with respect to $O^{\prime}$ is in block diagonal form, with a block corresponding to each (normalized) graded Jordan chain in $O$, and with the block corresponding to $\left(v_{1}^{\prime}, \ldots, v_{h}^{\prime}\right)$ above being an upper triangular matrix with superdiagonal $\left(\alpha_{1}, \ldots, \alpha_{h-1}\right)$ and 0 's elsewhere. Thus, for $0 \leqslant u \leqslant h-1$, we have

$$
D\left(v_{u+1}^{\prime}\right)=\alpha_{u} v_{u}^{\prime} \text {. }
$$

It follows that the subspace spanned by each graded Jordan chain in $O$ is closed under $U$ and $D$.

The Boolean algebra is the rank- $n$ graded poset obtained by partially ordering $B(n)$ by inclusion. The rank of a subset is given by cardinality.

Given $a=\left(a_{1}, \ldots, a_{n}\right), b=\left(b_{1}, \ldots, b_{n}\right) \in B_{X}(n)$, define $a \leqslant b$ provided $S(a) \subseteq S(b)$ and $a_{i}=b_{i}$ for all $i \in S(a)$. It is easy to see that this makes $B_{X}(n)$ into a rank- $n$ graded poset with rank of $a$ given by $|S(a)|$. We call $B_{X}(n)$ a generalized Boolean algebra. Clearly, when $|X|=1, B_{X}(n)$ is order isomorphic to $B(n)$. When $G$ is the group of all permutations of $X$ we have two orbits for the action on $G$ on $X \times X$ (i.e., $m=1$ ) and we call $B_{X}(n)$ the nonbinary analog of the Boolean algebra. We use $U_{n}$ to denote the up operator on both the posets $V(B(n))$ and $V\left(B_{X}(n)\right)$. Note that the action of $G \sim S_{n}$ on $B_{X}(n)$ is order and rank preserving and that $U_{n}$ is $G \sim S_{n}$-linear. Also note that the inner product on $V\left(B_{X}(n)\right)$ is $G \sim S_{n}$-invariant.

Consider the permutation representaion of $G$ on $V(X)$. A linear map $f: V(X) \rightarrow$ $V(X)$ is $G$-linear iff the $X \times X$ matrix $M_{f}$ representing $f$ (in the standard basis $X$ ) is constant on the orbits of the action of $G$ on $X \times X$. It follows that $\operatorname{dim} \operatorname{End}_{G}(V(X))=$ $m+1$.

Consider the canonical orthogonal decomposition

$$
V(X)=W_{0} \oplus \cdots \oplus W_{m},
$$


of $V(X)$ into distinct irreducible $G$-submodules, where $W_{0}$ corresponds to the trivial representation. Set $d_{i}=\operatorname{dim} W_{i}, i=0,1, \ldots, m$, so $d_{0}=1$ and $d_{0}+\cdots+d_{m}=|X|$.

For $u=0, \ldots, m$, define the linear map $f_{u}: V(X) \rightarrow V(X)$ by

$$
f_{u}(y)=\sum x, \quad y \in X
$$

where the sum is over all $x \in X$ with $(x, y) \in Z_{u}$. The matrix of $f_{u}$, in the standard basis $X$, is the $0-1$ matrix with a 1 in position $(x, y)$ iff $(x, y) \in Z_{u}$. Thus $f_{u}$ is $G$-linear. Since $W_{0}, \ldots, W_{m}$ are distinct irreducibles we have by Schur's lemma that each of $W_{0}, \ldots, W_{m}$ is $f_{u}$ invariant for each $u$ and that the action of each $f_{u}$ on each of $W_{0}, \ldots, W_{m}$ is multiplication by a scalar. For $u, w=0, \ldots, m$, let the action of $f_{u}$ on $W_{w}$ be multiplication by the scalar $\lambda(u, w)$.

Set

$$
\begin{aligned}
\mathcal{J}_{X}(n) & =\left\{(k, s, p): 0 \leqslant k \leqslant n, k^{-} \leqslant s \leqslant k, p \in C(s, m)\right\} \\
& =\left\{(k, s, p): 0 \leqslant s \leqslant n, s \leqslant k \leqslant\left\lfloor\frac{n+s}{2}\right\rfloor, p \in C(s, m)\right\} .
\end{aligned}
$$

For $(k, s, p) \in \mathcal{J}_{X}(n)$ with $p=\left(p_{1}, \ldots, p_{m}\right)$ define

$$
\mu(n, k, s, p)=\left(\begin{array}{c}
n \\
n-s, p_{1}, \ldots, p_{m}
\end{array}\right) d_{1}^{p_{1}} \ldots d_{m}^{p_{m}}\left\{\left(\begin{array}{c}
n-s \\
k-s
\end{array}\right)-\left(\begin{array}{c}
n-s \\
k-s-1
\end{array}\right)\right\} .
$$

(We take the multinomial coefficient $\left(\begin{array}{c}n \\ k_{1}, \ldots, k_{r}\end{array}\right)$ to be zero if any of $n, k_{1}, \ldots, k_{r}$ is negative or if $\left.n \neq k_{1}+\cdots+k_{r}\right)$.

The following two definitions are from [8] and [2] respectively.

For $i, j, k, t \in\{0, \ldots, n\}$ define

$$
\beta_{i, j, k}^{n, t}=\sum_{u=0}^{n}(-1)^{u-t}\left(\begin{array}{l}
u \\
t
\end{array}\right)\left(\begin{array}{c}
n-2 k \\
u-k
\end{array}\right)\left(\begin{array}{c}
n-k-u \\
i-u
\end{array}\right)\left(\begin{array}{c}
n-k-u \\
j-u
\end{array}\right) .
$$

For $l=\left(l_{0}, \ldots, l_{m}\right), p=\left(p_{0}, \ldots, p_{m}\right) \in \mathbb{Z}^{m+1}$ define

$$
\Lambda(\lambda, l, p)=\sum\left\{\prod_{w=0}^{m}\left(\begin{array}{c}
p_{w} \\
r(0, w), \ldots, r(m, w)
\end{array}\right)\right\}\left\{\prod_{u=0}^{m} \prod_{w=0}^{m} \lambda(u, w)^{r(u, w)}\right\},
$$

where the sum is over all $\{0, \ldots, m\} \times\{0, \ldots, m\}$ nonnegative integer matrices $(r(u, w))$ with row sums $l_{0}, \ldots, l_{m}$ and column sums $p_{0}, \ldots, p_{m}$. We take the empty sum to be zero.

For $i, j, t, k, s \in\{0, \ldots, n\}, l \in C(t, m+1)$, and $p=\left(p_{1}, \ldots, p_{m}\right) \in C(s, m)$, set $p_{0}=t-s, p^{+}=\left(p_{0}, p_{1}, \ldots, p_{m}\right)$ and define

$$
\alpha_{i, j, k, s}^{n, t, l, p}=(|X|)^{\frac{i+j}{2}-t} \Lambda\left(\lambda, l, p^{+}\right) \beta_{i-s, j-s, k-s}^{n-s, t-s} .
$$

For $0 \leqslant k \leqslant n, k^{-} \leqslant s \leqslant k$, and $k \leqslant i, j \leqslant n+s-k$, define $E_{i, j, k, s}$ to be the $\{k, k+1, \ldots, n+s-k\} \times\{k, k+1, \ldots, n+s-k\}$ matrix with entry in row $i$ and column $j$ equal to 1 and all other entries 0 .

We now state our result. Part (i) is proved in Lemma 3.2, part (ii) is proved in Theorem 3.3, and parts (iii) and (iv) are proved in Section 4. 
Theorem 1.1. Let $G$ be a finite group acting on the finite set $X$ such that the corresponding complex permutation representation is multiplicity free with $m+1$ distinct irreducibles occuring in it. Assume $m \geqslant 1$. Then

(i) There exists an orthogonal semisymmetric Jordan basis $J_{X}(n)$ of $V\left(B_{X}(n)\right)$.

(ii) $J_{X}(n)$ can be expressed as a disjoint union

$$
J_{X}(n)=\cup_{(k, s, p) \in \mathcal{J}_{X}(n)} J_{X}(n, k, s, p),
$$

where $J_{X}(n, k, s, p)$ consists of $\mu(n, k, s, p)$ semisymmetric Jordan chains starting at rank $k$ and ending at rank $n+s-k$.

Let $J_{X}^{\prime}(n)$ denote the orthonormal basis obtained by normalizing the vectors in $J_{X}(n)$. Define a $B_{X}(n) \times J_{X}^{\prime}(n)$ unitary matrix $M(n)$ as follows: for $v \in J_{X}^{\prime}(n)$, the column of $M(n)$ indexed by $v$ is the coordinate vector of $v$ in the standard basis $B_{X}(n)$.

(iii) $M(n)^{*} \mathcal{A}_{X}(n) M(n)$ consists of all $J_{X}^{\prime}(n) \times J_{X}^{\prime}(n)$ block diagonal matrices with a block corresponding to each (normalized) SSJC in $J_{X}(n)$ and such that, for each $(k, s, p) \in$ $\mathcal{J}_{X}(n)$, the $\mu(n, k, s, p)$ blocks corresponding to the SSJC's in $J_{X}(n, k, s, p)$ are identical.

(iv) Conjugating by $M(n)$ and, for each $(k, s, p) \in \mathcal{J}_{X}(n)$, preserving only one among the duplicate blocks corresponding to the SSJC's in $J_{X}(n, k, s, p)$, thus gives a *-algebra isomorphism

$$
\Phi: \mathcal{A}_{X}(n) \cong \bigoplus_{(k, s, p) \in \mathcal{J}_{X}(n)} \operatorname{Mat}((n+s-2 k+1) \times(n+s-2 k+1)) .
$$

It will be convenient to re-index the rows and columns of a block corresponding to a SSJC starting at rank $k$ and having offset $s$ by the set $\{k, k+1, \ldots, n+s-k\}$.

Let $(i, j, t, l) \in \mathcal{I}_{X}(n)$. Write

$$
\Phi\left(M_{i, j}^{t, l}\right)=\left(N_{k, s, p}\right),(k, s, p) \in \mathcal{J}_{X}(n) .
$$

Then

$$
N_{k, s, p}= \begin{cases}\left(\begin{array}{c}
n+s-2 k \\
i-k
\end{array}\right)^{-\frac{1}{2}}\left(\begin{array}{c}
n+s-2 k \\
j-k
\end{array}\right)^{-\frac{1}{2}} \alpha_{i, j, k, s}^{n, t, l, p} E_{i, j, k, s} & \text { if } k \leqslant i, j \leqslant n+s-k \\
0 & \text { otherwise. }\end{cases}
$$

\section{Symmetric group action on Boolean algebras}

In this section we summarize results on the $S_{n}$ action on $B(n)$ in the form needed in this paper. The main sources are Dunkl [5] and Schrijver [8]. For $0 \leqslant i, j, t \leqslant n$ let $M_{i, j}^{t}$ be the $B(n) \times B(n)$ matrix given by

$$
M_{i, j}^{t}(a, b)= \begin{cases}1 & \text { if }|a|=i,|b|=j,|a \cap b|=t \\ 0 & \text { otherwise. }\end{cases}
$$

It is clear that $\left\{M_{i, j}^{t}: i, j, t \in \mathbb{N}, t \leqslant i, t \leqslant j, i+j-t \leqslant n\right\}$ is a basis of

$$
\mathcal{A}(n)=\operatorname{End}_{S_{n}}(V(B(n))) \text {. }
$$


For $0 \leqslant k \leqslant n / 2$, and $k \leqslant i, j \leqslant n-k$, define $E_{i, j, k}$, to be the $\{k, k+1, \ldots, n-k\} \times$ $\{k, k+1, \ldots, n-k\}$ matrix with entry in row $i$ and column $j$ equal to 1 and all other entries 0 .

The following formulation is motivated by the one given in [8] (also see [9]). We do not use part (v) (a classical result of Delsarte [4]) in this paper but we have included it for completeness.

Theorem 2.1. (i) There exists an orthogonal $S J B J(n)$ of $V(B(n))$.

For $0 \leqslant k \leqslant n / 2$, let $J(n, k)$ be the union of all $S J C$ 's in $J(n)$ starting at rank $k$. For $0 \leqslant i \leqslant n$ and $0 \leqslant k \leqslant \min \{i, n-i\}$ set

$$
J(n, i, k)=\{v \in J(n, k): r(v)=i\}
$$

and let $V(n, i, k)$ be the subspace of $V\left(B(n)_{i}\right)$ spanned by $J(n, i, k)$.

(ii) Let $0 \leqslant i \leqslant n$. Then $V\left(B(n)_{i}\right)$ is a multiplicity free $S_{n}$-module and

$$
V\left(B(n)_{i}\right)=\bigoplus_{0 \leqslant k \leqslant \min \{i, n-i\}} V(n, i, k)
$$

is the orthogonal decomposition of $V\left(B(n)_{i}\right)$ into distinct irreducible submodules.

Let $J^{\prime}(n)$ denote the orthonormal basis of $V(B(n))$ obtained by normalizing $J(n)$. Define a $B(n) \times J^{\prime}(n)$ unitary matrix $N(n)$ as follows: for $v \in J^{\prime}(n)$, the column of $N(n)$ indexed by $v$ is the coordinate vector of $v$ in the standard basis $B(n)$.

(iii) $N(n)^{*} \mathcal{A}(n) N(n)$ consists of all $J^{\prime}(n) \times J^{\prime}(n)$ block diagonal matrices with a block corresponding to each (normalized) SJC in $J(n)$ and such that any two SJC's starting and ending at the same rank give rise to identical blocks. Thus there are $\left(\begin{array}{l}n \\ k\end{array}\right)-\left(\begin{array}{c}n \\ k-1\end{array}\right)$ identical blocks of size $(n-2 k+1) \times(n-2 k+1)$, for $k=0, \ldots,\lfloor n / 2\rfloor$.

(iv) Conjugating by $N(n)$ and dropping duplicate blocks thus gives a $*$-algebra isomorphism

$$
\Phi: \mathcal{A}(n) \cong \bigoplus_{k=0}^{\lfloor n / 2\rfloor} \operatorname{Mat}((n-2 k+1) \times(n-2 k+1)) .
$$

It will be convenient to re-index the rows and columns of a block corresponding to a SJC starting at rank $k$ and ending at rank $n-k$ by the set $\{k, k+1, \ldots, n-k\}$.

Let $i, j, t \in \mathbb{N}, t \leqslant i, t \leqslant j, i+j-t \leqslant n$. Write

$$
\Phi\left(M_{i, j}^{t}\right)=\left(N_{0}, \ldots, N_{\lfloor n / 2\rfloor}\right) .
$$

Then, for $0 \leqslant k \leqslant\lfloor n / 2\rfloor$,

$$
N_{k}= \begin{cases}\left(\begin{array}{c}
n-2 k \\
i-k
\end{array}\right)^{-\frac{1}{2}}\left(\begin{array}{c}
n-2 k \\
j-k
\end{array}\right)^{-\frac{1}{2}} \beta_{i, j, k}^{n, t} E_{i, j, k} & \text { if } k \leqslant i, j \leqslant n-k, \\
0 & \text { otherwise, }\end{cases}
$$

where $E_{i, j, k}=E_{i, j, k, 0}$. 
(v) Let $0 \leqslant i \leqslant n, i^{-} \leqslant t \leqslant i$, and $0 \leqslant k \leqslant \min \{i, n-i\}$. Substituting $j=i$ in part (iv) and simplifying we get that the eigenvalue of $M_{i, i}^{t}$ on $V(n, i, k)$ is

$$
\sum_{u=0}^{n}(-1)^{u-t}\left(\begin{array}{l}
u \\
t
\end{array}\right)\left(\begin{array}{c}
n-k-u \\
i-u
\end{array}\right)\left(\begin{array}{c}
i-k \\
i-u
\end{array}\right) .
$$

For a subspace analog (or $q$-analog) of Theorem 2.1, see $[\mathbf{1}, \mathbf{1 0}]$. For explicit constructions of orthogonal SJB's of $V(B(n))$ and $V\left(B_{q}(n)\right)$ (the subspace analog of $\left.V(B(n))\right)$ see $[9,11]$.

\section{Upper Boolean decomposition}

Let $(V, f)$ be a pair consisting of a finite dimensional inner product space $V$ (over $\mathbb{C}$ ) and a linear operator $f$ on $V$. Let $(W, g)$ be another such pair. By an isomorphism of pairs $(V, f)$ and $(W, g)$ we mean a linear isometry (i.e, an inner product preserving isomorphism) $\theta: V \rightarrow W$ such that $\theta(f(v))=g(\theta(v)), v \in V$.

Consider the inner product space $V\left(B_{X}(n)\right)$. An upper Boolean subspace of rank $t$ is a homogeneous subspace $W \subseteq V\left(B_{X}(n)\right)$ such that $\operatorname{rankset}(W)=\{n-t, n-t+$ $1, \ldots, n\}, W$ is closed under the up operator $U_{n}$, and there is an isomorphism of pairs $\left(V(B(t)), \sqrt{|X|} U_{t}\right) \cong\left(W, U_{n}\right)$ that sends homogeneous elements to homogeneous elements and increases rank by $n-t$.

Consider the identity

$$
\begin{aligned}
(|X|+1)^{n} & =\left(d_{1}+d_{2}+\cdots+d_{m}+2\right)^{n} \\
& =\sum_{\left(l_{0}, \ldots, l_{m}\right) \in C(n, m+1)}\left(\begin{array}{c}
n \\
l_{0}, \ldots, l_{m}
\end{array}\right) d_{1}^{l_{1}} d_{2}^{l_{2}} \cdots d_{m}^{l_{m}} 2^{l_{0}} .
\end{aligned}
$$

We shall now give a linear algebraic interpretation to the identity above.

Consider the inner product space $V(Y)$, with $Y$ as an orthonormal basis. Make the tensor product

$$
\otimes_{i=1}^{n} V(Y)=V(Y) \otimes \cdots \otimes V(Y) \quad(n \text { factors })
$$

into an inner product space by defining

$$
\left\langle v_{1} \otimes \cdots \otimes v_{n}, u_{1} \otimes \cdots \otimes u_{n}\right\rangle=\left\langle v_{1}, u_{1}\right\rangle \cdots\left\langle v_{n}, u_{n}\right\rangle .
$$

There is an isometry

$$
V\left(B_{X}(n)\right) \cong \otimes_{i=1}^{n} V(Y)
$$

given by $a=\left(a_{1}, \ldots, a_{n}\right) \mapsto \bar{a}=a_{1} \otimes \cdots \otimes a_{n}, a \in B_{X}(n)$. The rank function (on nonzero homogeneous elements) and the up and down operators, $U_{n}$ and $D_{n}$, on $V\left(B_{X}(n)\right)$ are transferred to $\otimes_{i=1}^{n} V(Y)$ via the isomorphism above. From now onwards, we shall not distinguish between $V\left(B_{X}(n)\right)$ and $\otimes_{i=1}^{n} V(Y)$. 
Choose orthonormal bases $\mathcal{B}_{i}$ for $W_{i}, i=0, \ldots, m$ and set $\mathcal{B}=\mathcal{B}_{0} \cup \cdots \cup \mathcal{B}_{m}$. We assume that $\mathcal{B}_{0}$ consists of the vector

$$
\frac{1}{\sqrt{|X|}} \sum_{x \in X} x=z .
$$

Note that orthonormality of $\mathcal{B}$ implies that if $v=\sum_{x \in X} \alpha_{x} x \in \mathcal{B}_{i}, i=1, \ldots, m$, then $\sum_{x \in X} \alpha_{x}=0$.

We have in $V(Y)$

$$
\begin{aligned}
& U_{1}(v)=D_{1}(v)=0, v \in \mathcal{B}_{i}, i=1, \ldots, m, \\
& U_{1}(z)=D_{1}\left(L_{0}\right)=0, \\
& U_{1}\left(L_{0}\right)=\sqrt{|X|} z, D_{1}(z)=\sqrt{|X|} L_{0} .
\end{aligned}
$$

Given $l=\left(l_{0}, \ldots, l_{m}\right) \in C(n, m+1)$ set

$$
\begin{aligned}
& S(n, m, l)= \\
& \quad\left\{\left(A_{1}, \ldots, A_{m}\right): A_{i} \subseteq[n] \text { for all } i, A_{i} \cap A_{j}=\emptyset \text { for } i \neq j, \text { and }\left|A_{i}\right|=l_{i} \text { for all } i\right\} .
\end{aligned}
$$

Given $\mathcal{A}=\left(A_{1}, \ldots, A_{m}\right) \in S(n, m, l)$ we set

$$
\begin{aligned}
\Sigma(\mathcal{A}) & =A_{1} \cup \cdots \cup A_{m}, \\
\mathcal{M}(\mathcal{A}) & =\left\{f=\left(f_{1}, \ldots, f_{m}\right) \mid f_{i}: A_{i} \rightarrow \mathcal{B}_{i} \text { for all } i\right\} .
\end{aligned}
$$

Given $l=\left(l_{0}, \ldots, l_{m}\right) \in C(n, m+1)$ define

$$
\begin{aligned}
\mathcal{S}_{X}(n, l) & =\{(\mathcal{A}, f): \mathcal{A} \in S(n, m, l), f \in \mathcal{M}(\mathcal{A})\} \\
\mathcal{K}_{X}(n, l) & =\{(\mathcal{A}, f, B): \mathcal{A} \in S(n, m, l), f \in \mathcal{M}(\mathcal{A}), B \subseteq[n]-\Sigma(\mathcal{A})\}
\end{aligned}
$$

Note that

$$
\begin{aligned}
\left|\mathcal{S}_{X}(n, l)\right| & =\left(\begin{array}{c}
n \\
l_{0}, \ldots, l_{m}
\end{array}\right) d_{1}^{l_{1}} d_{2}^{l_{2}} \cdots d_{m}^{l_{m}} \\
\left|\mathcal{K}_{X}(n, l)\right| & =\left(\begin{array}{c}
n \\
l_{0}, \ldots, l_{m}
\end{array}\right) d_{1}^{l_{1}} d_{2}^{l_{2}} \cdots d_{m}^{l_{m}} 2^{l_{0}} .
\end{aligned}
$$

For $(\mathcal{A}, f, B) \in \mathcal{K}_{X}(n, l)$, with $\mathcal{A}=\left(A_{1}, \ldots, A_{m}\right), f=\left(f_{1}, \ldots, f_{m}\right)$, define a vector

$$
v(\mathcal{A}, f, B)=v_{1} \otimes \cdots \otimes v_{n} \in \otimes_{i=1}^{n} V(Y)
$$

by

$$
v_{i}= \begin{cases}f_{j}(i) & \text { if } i \in A_{j}, \\ z & \text { if } i \in B, \\ L_{0} & \text { if } i \in[n]-(\Sigma(\mathcal{A}) \cup B) .\end{cases}
$$


Note that $v(\mathcal{A}, f, B)$ is a homogeneous vector in $\otimes_{i=1}^{n} V(Y)$ of $\operatorname{rank}|\Sigma(\mathcal{A})|+|B|$.

Given $l \in C(n, m+1)$ and $(\mathcal{A}, f) \in \mathcal{S}_{X}(n, l)$, set

$$
K_{X}(l, \mathcal{A}, f)=\{v(\mathcal{A}, f, B): B \subseteq([n]-\Sigma(\mathcal{A}))\}
$$

and define $V_{(l, \mathcal{A}, f)}$ to be the subspace of $\otimes_{i=1}^{n} V(Y)$ spanned by $K_{X}(l, \mathcal{A}, f)$. Set

$$
K_{X}(n)=\cup_{l} \cup_{(\mathcal{A}, f)} K_{X}(l, \mathcal{A}, f),
$$

where the union is over $l \in C(n, m+1)$ and $(\mathcal{A}, f) \in \mathcal{S}_{X}(n, l)$.

We have, using (13), (14), and (15), the following formula in $\otimes_{i=1}^{n} V(Y)$ : for $v(\mathcal{A}, f, B) \in K_{X}(n)$

$$
U_{n}(v(\mathcal{A}, f, B))=\sqrt{|X|}\left\{\sum_{B^{\prime}} v\left(\mathcal{A}, f, B^{\prime}\right)\right\},
$$

where the sum is over all $B^{\prime} \subseteq([n]-\Sigma(\mathcal{A}))$ covering $B$.

It follows from the orthonormality of $\mathcal{B}$ and the inner product formula (11) that

$$
\left\langle v(\mathcal{A}, f, B), v\left(\mathcal{A}^{\prime}, f^{\prime}, B^{\prime}\right)\right\rangle=\delta\left((\mathcal{A}, f, B),\left(\mathcal{A}^{\prime}, f^{\prime}, B^{\prime}\right)\right)
$$

where $v(\mathcal{A}, f, B), v\left(\mathcal{A}^{\prime}, f^{\prime}, B^{\prime}\right) \in K_{X}(n)$ and $\delta$ is the Kronecker delta.

We can summarize the discussion above in the following result.

Theorem 3.1. (i) For $l \in C(n, m+1),(\mathcal{A}, f) \in \mathcal{S}_{X}(n, l), V_{(l, \mathcal{A}, f)}$ is an upper Boolean subspace of $\otimes_{i=1}^{n} V(Y)$ of rank $n-|\Sigma(\mathcal{A})|$ and with orthonormal basis $K_{X}(l, \mathcal{A}, f)$.

(ii) $K_{X}(n)$ is an orthonormal basis of $\otimes_{i=1}^{n} V(Y)$.

(iii) We have the following orthogonal decomposition into upper Boolean subspaces:

$$
\bigotimes_{i=1}^{n} V(Y)=\bigoplus_{l} \bigoplus_{\mathcal{A}} \bigoplus_{f} V_{(l, \mathcal{A}, f)}
$$

where the sum is over all $l \in C(n, m+1), \mathcal{A} \in S(n, m, l)$ and $f \in \mathcal{M}(\mathcal{A})$.

For each $\left(l_{0}, \ldots, l_{m}\right) \in C(n, m+1)$ the right hand side of (20) contains

$$
\left(\begin{array}{c}
n \\
l_{0}, \ldots, l_{m}
\end{array}\right) d_{1}^{l_{1}} d_{2}^{l_{2}} \cdots d_{m}^{l_{m}}
$$

upper Boolean subspaces of rank $l_{0}$.

Taking dimensions on both sides of (20) yields a linear algebraic interpretation to identity (10) above. Certain problems on the generalized Boolean algebra $B_{X}(n)$ can be reduced to corresponding problems on the Boolean algebra $B(n)$ via the basis $K_{X}(n)$. 
Lemma 3.2. (i) For $l=\left(l_{0}, \ldots, l_{m}\right) \in C(n, m+1),(\mathcal{A}, f) \in \mathcal{S}_{X}(n, l)$ there exists an orthogonal $S S J B J_{X}(l, \mathcal{A}, f)$ of $V_{(l, \mathcal{A}, f)}$, i.e., there exists a graded Jordan basis $J_{X}(l, \mathcal{A}, f)$ of $V_{(l, \mathcal{A}, f)}$ (with respect to the up operator $U_{n}$ ) such that each graded Jordan chain in $J_{X}(l, \mathcal{A}, f)$ is a SSJC in $\bigotimes_{i=1}^{n} V(Y)$.

Each $S S J C$ in $J_{X}(l, \mathcal{A}, f)$ has offset $s=l_{1}+\cdots+l_{m}$. For $s \leqslant k \leqslant\left\lfloor\frac{n+s}{2}\right\rfloor$, denote by $J_{X}(k, l, \mathcal{A}, f)$ the set of all $S S J C$ 's in $J_{X}(l, \mathcal{A}, f)$ starting at rank $k$. Then

$$
\left|J_{X}(k, l, \mathcal{A}, f)\right|=\left(\begin{array}{l}
n-s \\
k-s
\end{array}\right)-\left(\begin{array}{c}
n-s \\
k-s-1
\end{array}\right) .
$$

(ii) $J_{X}(n)=\cup J_{X}(l, \mathcal{A}, f)$, where the union is over all $l \in C(n, m+1)$ and $(\mathcal{A}, f) \in$ $\mathcal{S}_{X}(n, l)$, is an orthogonal SSJB of $\otimes_{i=1}^{n} V(Y)$.

Proof. (i) Consider the upper Boolean subspace $V_{(l, \mathcal{A}, f)}$ of rank $l_{0}$. Let $\gamma:\left\{1,2, \ldots, l_{0}\right\} \rightarrow$ $[n]-\Sigma(\mathcal{A})$ be the unique order preserving bijection, i.e., $\gamma(i)=i^{t h}$ smallest element of $[n]-\Sigma(\mathcal{A})$. Denote by $\gamma^{\prime}: V\left(B\left(l_{0}\right)\right) \rightarrow V_{(l, \mathcal{A}, f)}$ the linear isometry given by $\gamma^{\prime}(X)=$ $v(\mathcal{A}, f, \gamma(X)), X \in B\left(l_{0}\right)$.

Use Theorem 2.1 (i) to get an orthogonal SJB $J\left(l_{0}\right)$ of $V\left(B\left(l_{0}\right)\right)$ with respect to $\sqrt{|X|} U_{l_{0}}$ (rather than just $U_{l_{0}}$ ) and transfer it to $V_{(l, \mathcal{A}, f)}$ via $\gamma^{\prime}$. Each SJC in $J\left(l_{0}\right)$ will get transferred to a SSJC in $V_{(l, \mathcal{A}, f)}$ of offset $s$. The number of these SSJC's in $V_{(l, \mathcal{A}, f)}$ starting at rank $k$ is $\left(\begin{array}{l}n-s \\ k-s\end{array}\right)-\left(\begin{array}{c}n-s \\ k-s-1\end{array}\right)$.

(ii) This is clear.

We shall now make three observations on the action of $G \sim S_{n}$ on the basis elements in $J_{X}(k, l, \mathcal{A}, f)$.

Let $l=\left(l_{0}, l_{1}, \ldots, l_{m}\right) \in C(n, m+1)$ and $(\mathcal{A}, f) \in \mathcal{S}_{X}(n, l)$. Write $\mathcal{A}=\left(A_{1}, \ldots, A_{m}\right)$ and $f=\left(f_{1}, \ldots, f_{m}\right)$.

(a) Let $v \in J_{X}(k, l, \mathcal{A}, f)$ (i.e., $v$ is a vector lying in one of the $\operatorname{SSJC}$ 's in $J_{X}(l, \mathcal{A}, f)$ starting at rank $k$ ) of rank $i$ and let $\tau=(1, \ldots, 1, \pi) \in G \sim S_{n}(1=$ identity in $G)$.

Write $\pi \mathcal{A}=\left(\pi A_{1}, \ldots, \pi A_{m}\right)=\mathcal{A}^{\prime}$ and $f \pi^{-1}=\left(\left.f_{1} \pi^{-1}\right|_{\pi A_{1}}, \ldots,\left.f_{m} \pi^{-1}\right|_{\pi A_{m}}\right)=f^{\prime}$. We want to show that $\tau v$ is in the subspace spanned by all elements of $J_{X}\left(k, l, \mathcal{A}^{\prime}, f^{\prime}\right)$ of rank $i$.

Let $\sigma:[n]-\Sigma(\mathcal{A}) \rightarrow[n]-\Sigma\left(\mathcal{A}^{\prime}\right)$ be the unique order preserving bijection. There is an obvious isomorphism of pairs $\Gamma:\left(V_{(l, \mathcal{A}, f)}, U_{n}\right) \rightarrow\left(V_{\left(l, \mathcal{A}^{\prime}, f^{\prime}\right)}, U_{n}\right)$ taking $v(\mathcal{A}, f, B) \in V_{(l, \mathcal{A}, f)}$ to $\Gamma(v(\mathcal{A}, f, B))=v\left(\mathcal{A}^{\prime}, f^{\prime}, \sigma B\right)$.

Clearly we have $\Gamma(v) \in J_{X}\left(k, l, \mathcal{A}^{\prime}, f^{\prime}\right)$ with $r(\Gamma(v))=i$. Define a permutation $\pi^{\prime}$ : $[n] \rightarrow[n]$ as follows: $\pi^{\prime}(i)=i$ for $i \in \Sigma\left(\mathcal{A}^{\prime}\right)$ and $\pi^{\prime}(i)=\pi \sigma^{-1}(i)$ for $i \in[n]-\Sigma\left(\mathcal{A}^{\prime}\right)$. Set $\tau^{\prime}=\left(1, \ldots, 1, \pi^{\prime}\right) \in G \sim S_{n}$. A little reflection shows that $\tau v=\tau^{\prime} \Gamma(v)$.

Now, by Theorem 2.1 (ii), the subspace spanned by all elements of $J_{X}\left(k, l, \mathcal{A}^{\prime}, f^{\prime}\right)$ of rank $i$ is closed under permutations of $[n]$ that fix $\Sigma\left(\mathcal{A}^{\prime}\right)$ and thus it follows that $\tau v$ is in this subspace.

(b) Let $v \in J_{X}(k, l, \mathcal{A}, f), i \in A_{j}, g \in G$, and $\tau=(1, \ldots, 1, g, 1, \ldots, 1$, id $) \in G \sim S_{n}$, with $g$ in the $i$ th spot and id denoting the identity of $S_{n}$. Then we have

$$
\tau v=\sum_{e} \alpha_{e} v_{e}
$$


where the $\alpha_{e}$ are scalars, $v_{e} \in J_{X}(k, l, \mathcal{A}, e)$, and where the sum is over all

$$
e=\left(f_{1}, \ldots, f_{j-1}, e_{j}, f_{j+1}, \ldots, f_{m}\right)
$$

with $e_{j}: A_{j} \rightarrow \mathcal{B}_{j}$ satisfying $e_{j}(w)=f_{j}(w)$, for all $w \in A_{j}-\{i\}$.

(c) Let $v \in J_{X}(k, l, \mathcal{A}, f), i \in[n]-\Sigma(\mathcal{A}), g \in G$, and $\tau=(1, \ldots, 1, g, 1, \ldots, 1$, id $) \in$ $G \sim S_{n}$, with $g$ in the $i$ th spot and id denoting the identity of $S_{n}$. Then we have $\tau v=v$.

Theorem 3.3. Let $0 \leqslant s \leqslant n, p=\left(p_{1}, \ldots, p_{m}\right) \in C(s, m)$ and set $l=\left(n-s, p_{1}, \ldots, p_{m}\right) \in$ $C(n, m+1)$.

Define

$$
V_{(s, p)}=\bigoplus_{\mathcal{A}} \bigoplus_{f} V_{(l, \mathcal{A}, f)}, \quad J_{X}(n, s, p)=\cup_{(\mathcal{A}, f)} J_{X}(l, \mathcal{A}, f)
$$

where the sum and the disjoint union are over all $(\mathcal{A}, f) \in \mathcal{S}_{X}(n, l)$.

(i) We have the following orthogonal decomposition

$$
\bigotimes_{i=1}^{n} V(Y)=\bigoplus_{0 \leqslant s \leqslant n} \bigoplus_{p \in C(s, m)} V_{(s, p)} .
$$

(ii) $J_{X}(n, s, p)$ is an orthogonal SSJB of $V_{(s, p)}$ with each SSJC in $J_{X}(n, s, p)$ having offset $s$. For $s \leqslant k \leqslant\left\lfloor\frac{n+s}{2}\right\rfloor$ define $J_{X}(n, k, s, p)$ to be the collection of all chains in $J_{X}(n, s, p)$ starting at rank $k$. Then $J_{X}(n, k, s, p)$ consists of $\mu(n, k, s, p)$ semisymmetric Jordan chains starting at rank $k$ and ending at rank $n+s-k$. We have

$$
J_{X}(n)=\cup_{(k, s, p) \in \mathcal{J}_{X}(n)} J_{X}(n, k, s, p) .
$$

Proof. Clear.

For $0 \leqslant i \leqslant n$, define

$$
\begin{aligned}
\mathcal{J}_{X}(n, i) & =\left\{(k, s, p) \in \mathcal{J}_{X}(n): k \leqslant i \leqslant n+s-k\right\} \\
& =\left\{(k, s, p) \in \mathcal{J}_{X}(n): s \leqslant k \leqslant \min \{i, n+s-i\}\right\} .
\end{aligned}
$$

For $0 \leqslant i \leqslant n$ and $(k, s, p) \in \mathcal{J}_{X}(n, i)$, define

$$
J_{X}(n, i, k, s, p)=\left\{v \in J_{X}(n, k, s, p): r(v)=i\right\}
$$

and $V_{X}(n, i, k, s, p)$ to be the subspace of $V\left(B_{X}(n)_{i}\right)$ spanned by $J_{X}(n, i, k, s, p)$. We have the following orthogonal decomposition into subspaces

$$
V\left(B_{X}(n)_{i}\right)=\bigoplus_{(k, s, p) \in \mathcal{J}_{X}(n, i)} V_{X}(n, i, k, s, p) .
$$

Theorem 3.4. (Ceccherini-Silberstein, Scarabotti, and Tolli [2]) The action of $G \sim S_{n}$ on $V\left(B_{X}(n)_{i}\right)$ is multiplicity free and (22) gives the decomposition into distinct irreducible submodules. 
Proof. Let $0 \leqslant i \leqslant n$ and $(k, s, p) \in \mathcal{J}_{X}(n, i)$. It follows from the three properties (a), (b), and (c) stated above that $V_{X}(n, i, k, s, p)$ is a $G \sim S_{n}$-submodule of $\otimes_{i=1}^{n} V(Y)$.

The decomposition (22) is indexed by $\mathcal{J}_{X}(n, i)$ and a basis of $\operatorname{End}_{G \sim S_{n}}\left(V\left(B_{X}(n)_{i}\right)\right)$ is indexed by $\mathcal{I}_{X}(n, i)$. The result will follow if we show that $\mathcal{J}_{X}(n, i)$ and $\mathcal{I}_{X}(n, i)$ have the same cardinality.

Case (i) $i \leqslant n / 2$ : We have

$$
\begin{aligned}
\mathcal{I}_{X}(n, i) & =\{(t, l): 0 \leqslant t \leqslant i, l \in C(t, m+1)\} \\
\mathcal{J}_{X}(n, i) & =\{(k, s, p): 0 \leqslant s \leqslant k \leqslant i, p \in C(s, m)\} \\
& =\{(k, p): 0 \leqslant k \leqslant i, p \in C(k, m+1)\}
\end{aligned}
$$

Clearly $\mathcal{I}_{X}(n, i)$ and $\mathcal{J}_{X}(n, i)$ have the same cardinality.

Case (ii) $i>n / 2$ : We have

$$
\begin{aligned}
\mathcal{I}_{X}(n, i) & =\{(t, l): 2 i-n \leqslant t \leqslant i, l \in C(t, m+1)\} \\
& =\{(t, s, p): 2 i-n \leqslant t \leqslant i, 0 \leqslant s \leqslant t, p \in C(s, m)\} \\
& =\{(t, s, p): 0 \leqslant s, \quad \max \{2 i-n, s\} \leqslant t \leqslant i, p \in C(s, m)\} . \\
\mathcal{J}_{X}(n, i) & =\{(k, s, p): 0 \leqslant s, s \leqslant k \leqslant \min \{i, n+s-i\}, p \in C(s, m)\} .
\end{aligned}
$$

Clearly $\mathcal{I}_{X}(n, i)$ and $\mathcal{J}_{X}(n, i)$ have the same cardinality.

We have from Theorem 3.4 above that, for $0 \leqslant i \leqslant n$, the $V_{X}(n, i, k, s, p),(k, s, p) \in$ $\mathcal{J}_{X}(n, i)$ are the common eigenspaces for $M_{i, i}^{t, l},(t, l) \in \mathcal{I}_{X}(n, i)$. The eigenvalues will follow from part (iv) of Theorem 1.1 (see Theorem 4.4).

\section{Explicit block diagonalization}

We shall need the following result.

Lemma 4.1. For $m \geqslant 1$ we have

$$
\sum_{k=0}^{n} \sum_{s=k^{-}}^{k}(n+s-2 k+1)^{2}\left(\begin{array}{c}
s+m-1 \\
m-1
\end{array}\right)=\left(\begin{array}{c}
n+m+3 \\
m+3
\end{array}\right) .
$$

Proof. We shall use the following well known identities:

(i) For $n$ odd, $1^{2}+3^{2}+5^{2}+\cdots+n^{2}=\left(\begin{array}{c}n+2 \\ 3\end{array}\right)$.

(ii) For $n$ even, $2^{2}+4^{2}+6^{2}+\cdots+n^{2}=\left(\begin{array}{c}n+2 \\ 3\end{array}\right)$.

(iii) For $r, s \geqslant 0$ we have

$$
\sum_{k=0}^{n}\left(\begin{array}{c}
k+r \\
r
\end{array}\right)\left(\begin{array}{c}
n-k+s \\
s
\end{array}\right)=\left(\begin{array}{c}
n+r+s+1 \\
r+s+1
\end{array}\right) .
$$

(For the proof multiply the expansions $\sum_{k \geqslant 0}\left(\begin{array}{c}k+r \\ r\end{array}\right) x^{k}=\frac{1}{(1-x)^{r+1}}$ and $\sum_{k \geqslant 0}\left(\begin{array}{c}k+s \\ s\end{array}\right) x^{k}=$ $\frac{1}{(1-x)^{s+1}}$ and compare coefficients of $x^{n}$ on both sides.) 
We have

$$
\begin{aligned}
\sum_{s=0}^{n} & \sum_{k=s}^{\left\lfloor\frac{n+s}{2}\right\rfloor}(n+s-2 k+1)^{2}\left(\begin{array}{c}
s+m-1 \\
m-1
\end{array}\right) \\
= & \sum_{s=0, n+s \text { even }}^{n}\left(1^{2}+3^{2}+\cdots+(n-s+1)^{2}\right)\left(\begin{array}{c}
s+m-1 \\
m-1
\end{array}\right) \\
& +\sum_{s=0, n+s \text { odd }}^{n}\left(2^{2}+4^{2}+\cdots+(n-s+1)^{2}\right)\left(\begin{array}{c}
s+m-1 \\
m-1
\end{array}\right) \\
= & \sum_{s=0}^{n}\left(\begin{array}{c}
n-s+3 \\
3
\end{array}\right)\left(\begin{array}{c}
s+m-1 \\
m-1
\end{array}\right) \\
= & \left(\begin{array}{c}
n+m+3 \\
m+3
\end{array}\right) .
\end{aligned}
$$

Consider the linear operator on $V\left(B_{X}(n)\right)$ whose matrix with respect to the standard basis $B_{X}(n)$ is $M_{i, j}^{t, l}$. Transfer this operator to $\otimes_{i=1}^{n} V(Y)$ via the isomorphism (12) above and denote the resulting linear operator by $\mathcal{M}_{i, j}^{t, l}$. In Theorem 4.3 below we show that the action of $\mathcal{M}_{i, j}^{t, l}$ on the basis $K_{X}(n)$ mirrors the action of $M_{i, j}^{t}$ on the standard basis of the Boolean algebra $B(n)$.

Recall the maps $f_{u}$ on $V(X)$ from the introduction. Define linear operators $\mathcal{Z}, \mathcal{R}_{u}$ : $V(Y) \rightarrow V(Y), u=0, \ldots, m$, on $V(Y)$ as follows

- $\mathcal{Z}\left(L_{0}\right)=L_{0}$ and $\mathcal{Z}(x)=0$ for $x \in X$.

- For $u=0, \ldots, m, \mathcal{R}_{u}\left(L_{0}\right)=0$ and $\mathcal{R}_{u}(y)=f_{u}(y), y \in X$.

Note that, for $w=0, \ldots, m$,

$$
\mathcal{R}_{u}(v)=\lambda(u, w) v, v \in \mathcal{B}_{w} .
$$

Let there be given a $(m+4)$-tuple

$$
\mathcal{X}=\left(S_{U}, S_{D}, S_{\mathcal{Z}}, S_{\mathcal{R}_{0}}, \ldots, S_{\mathcal{R}_{m}}\right)
$$

of pairwise disjoint subsets of $[n]$ with union $[n]$ (it is convenient to index the components of $\mathcal{X}$ in this fashion). Define a linear operator

$$
F(\mathcal{X}): \bigotimes_{i=1}^{n} V(Y) \rightarrow \bigotimes_{i=1}^{n} V(Y)
$$

by $F(\mathcal{X})=F_{1} \otimes \cdots \otimes F_{n}$, where each $F_{i}$ is $U_{1}$ or $D_{1}$ or $\mathcal{Z}$ or $\mathcal{R}_{j}$ according as $i \in S_{U}$ or $S_{D}$ or $S_{\mathcal{Z}}$ or $S_{\mathcal{R}_{j}}$, respectively.

Let $b \in B_{X}(n)$. It follows from the definitions that

$$
F(\mathcal{X})(\bar{b}) \neq 0 \quad \text { implies } \quad S_{D} \cup S_{\mathcal{R}_{0}} \cup \cdots \cup S_{\mathcal{R}_{m}}=S(b), S_{U} \cup S_{\mathcal{Z}}=[n]-S(b) .
$$


Given a $(m+4)$-tuple $r=\left(r_{U}, r_{D}, r_{\mathcal{Z}}, r_{\mathcal{R}_{0}}, \ldots, r_{\mathcal{R}_{m}}\right) \in C(n, m+4)$ define $\Pi(r)$ to be the set of all $(m+4)$-tuples $\mathcal{X}=\left(S_{U}, S_{D}, S_{\mathcal{Z}}, S_{\mathcal{R}_{0}}, \ldots, S_{R_{m}}\right)$ of pairwise disjoint subsets of $[n]$ with union $[n]$ and with $\left|S_{U}\right|=r_{U},\left|S_{D}\right|=r_{D},\left|S_{\mathcal{Z}}\right|=r_{\mathcal{Z}}$, and $\left|S_{\mathcal{R}_{j}}\right|=r_{\mathcal{R}_{j}}$, for $j=0, \ldots, m$.

Lemma 4.2. Let $(i, j, t, l) \in \mathcal{I}_{X}(n)$ with $l=\left(l_{0}, \ldots, l_{m}\right)$. Set $r=(i-t, j-t, n+t-i-$ $\left.j, l_{0}, \ldots, l_{m}\right)$. Then

$$
\mathcal{M}_{i, j}^{t, l}=\sum_{\mathcal{X} \in \Pi(r)} F(\mathcal{X})
$$

Proof. Let $b=\left(b_{1}, \ldots, b_{n}\right) \in B_{X}(n)$ and $\mathcal{X}=\left(S_{U}, S_{D}, S_{\mathcal{Z}}, S_{\mathcal{R}_{0}}, \ldots, S_{\mathcal{R}_{m}}\right) \in \Pi(r)$. We consider two cases:

(i) $|S(b)| \neq j$ : In this case we have $\mathcal{M}_{i, j}^{t, l}(\bar{b})=0$. Now $\left|S_{D}\right|+\left|S_{\mathcal{R}_{0}}\right|+\cdots+\left|S_{\mathcal{R}_{m}}\right|=$ $j-t+l_{0}+\cdots+l_{m}=j$. Thus, from $(24)$, we also have $F(\mathcal{X})(\bar{b})=0$.

(ii) $|S(b)|=j$ : Assume that $F(\mathcal{X})(\bar{b}) \neq 0$. We have from $(24)$ that $F(\mathcal{X})(\bar{b})=\sum_{a} \bar{a}$, where the sum is over all $a=\left(a_{1}, \ldots, a_{n}\right) \in B_{X}(n)_{i}$ with $S(a)=S_{U} \cup S_{\mathcal{R}_{0}} \cup \cdots \cup S_{\mathcal{R}_{m}}$ and $\left(a_{k}, b_{k}\right) \in Z_{q}$, for $k \in S_{\mathcal{R}_{q}}, q=0, \ldots, m$.

Going over all elements of $\Pi(r)$ and summing we see that both sides of (25) evaluate to the same element on $\bar{b}$.

Theorem 4.3. Let $0 \leqslant s \leqslant n, p=\left(p_{1}, \ldots, p_{m}\right) \in C(s, m)$ and $(i, j, t, l) \in \mathcal{I}_{X}(n)$. Let $(\mathcal{A}, f, B) \in \mathcal{K}_{X}\left(n,\left(n-s, p_{1}, \ldots, p_{m}\right)\right)$. Set $p_{0}=t-s$ and $p^{+}=\left(p_{0}, p_{1}, \ldots, p_{m}\right)$.

(i) $\mathcal{M}_{i, j}^{t, l}(v(\mathcal{A}, f, B))=0$ if $|B| \neq j-s$.

(ii) If $|B|=j-s$ then

$$
\mathcal{M}_{i, j}^{t, l}(v(\mathcal{A}, f, B))=(|X|)^{\frac{i+j}{2}-t} \Lambda\left(\lambda, l, p^{+}\right)\left(\sum_{B^{\prime}} v\left(\mathcal{A}, f, B^{\prime}\right)\right),
$$

where the sum is over all $B^{\prime} \subseteq([n]-\Sigma(\mathcal{A}))$ with $\left|B^{\prime}\right|=i-s$ and $\left|B \cap B^{\prime}\right|=t-s$.

Proof. Let $r=\left(i-t, j-t, n+t-i-j, l_{0}, \ldots, l_{m}\right)$, where $l=\left(l_{0}, \ldots, l_{m}\right)$ and let $\mathcal{X}=\left(S_{U}, S_{D}, S_{\mathcal{Z}}, S_{\mathcal{R}_{0}}, \ldots, S_{\mathcal{R}_{m}}\right) \in \Pi(r)$. Assume that $F(\mathcal{X})(v(\mathcal{A}, f, B)) \neq 0$. Then we must have

$$
\begin{aligned}
S_{U} \cup S_{\mathcal{Z}} & =[n]-(\Sigma(\mathcal{A}) \cup B), \\
S_{D} \cup S_{\mathcal{R}_{0}} \cup \cdots \cup S_{\mathcal{R}_{m}} & =\Sigma(\mathcal{A}) \cup B, \\
S_{D} & \subseteq B .
\end{aligned}
$$

Thus, using (28) above, $|B|=j-t+l_{0}+\cdots+l_{m}-s=j-s$ (so part (i) follows).

We have

$$
S_{\mathcal{R}_{0}} \cup \cdots \cup S_{\mathcal{R}_{m}}=\Sigma(\mathcal{A}) \cup\left(B-S_{D}\right) .
$$

Since each vector in $\mathcal{B}$ is an eigenvector for each of $\mathcal{R}_{0}, \ldots, \mathcal{R}_{m}$ we see that

$$
F(\mathcal{X})(v(\mathcal{A}, f, B))=\alpha v\left(\mathcal{A}, f, B^{\prime}\right),
$$


where $\alpha$ is a scalar and where $B^{\prime}=S_{U} \cup\left(B-S_{D}\right)$, yielding $\left|B^{\prime}\right|=i-s$ and $\left|B \cap B^{\prime}\right|=$ $\left|B-S_{D}\right|=(j-s)-(j-t)=t-s$.

We now determine $\alpha$. Write $\mathcal{A}=\left(A_{1}, \ldots, A_{m}\right)$ and put $A_{0}=B-S_{D}$. It is easily seen, using (15) and the definition of $\lambda(u, w)$, that

$$
\alpha=|X|^{\frac{i+j}{2}-t} \prod_{u=0}^{m} \prod_{w=0}^{m} \lambda(u, w)^{\left|S_{\mathcal{R}_{u}} \cap A_{w}\right|} .
$$

The $\{0,1, \ldots, m\} \times\{0,1, \ldots, m\}$ integer matrix with entry in row $u$, column $w$ equal to $\left|S_{\mathcal{R}_{u}} \cap A_{w}\right|$ has row sums $l_{0}, \ldots, l_{m}$ and columns sums $p_{0}, p_{1}, \ldots, p_{m}$.

Now fix a $\{0,1, \ldots, m\} \times\{0,1, \ldots, m\}$ integer matrix $(r(u, w))$ with row sums $l_{0}, \ldots, l_{m}$ and columns sums $p_{0}, p_{1}, \ldots, p_{m}$ and fix $B^{\prime} \subseteq([n]-\Sigma(\mathcal{A}))$ with $\left|B^{\prime}\right|=i-s$ and $\left|B \cap B^{\prime}\right|=$ $t-s$. We want to count the number of $\mathcal{X}=\left(S_{U}, S_{D}, S_{\mathcal{Z}}, S_{\mathcal{R}_{0}}, \ldots, S_{\mathcal{R}_{m}}\right) \in \Pi(r)$ with $F(\mathcal{X})(v(\mathcal{A}, f, B))=\alpha v\left(\mathcal{A}, f, B^{\prime}\right)$, where $\left|S_{\mathcal{R}_{u}} \cap A_{w}\right|=r(u, w)$ for $u, w \in\{0, \ldots, m\}$ and $\alpha$ is given by (30) above.

The discussion above shows that $S_{U}=B^{\prime}-B$ and $S_{D}=B-B^{\prime}$ and (27) above then determines $S_{\mathcal{Z}}$. The number of choices for $S_{\mathcal{R}_{0}}, \ldots, S_{\mathcal{R}_{m}}$ is easily seen to be

$$
\left\{\prod_{w=0}^{m}\left(\begin{array}{c}
p_{w} \\
r(0, w), \ldots, r(m, w)
\end{array}\right)\right\} .
$$

Going over all elements of $\Pi(r)$ and summing we get the result.

Proof of Theorem 1.1 (parts (iii) and (iv)):

(iii) Let $(i, j, t, l) \in \mathcal{I}_{X}(n)$ and $(k, s, p) \in \mathcal{J}_{X}(n)$. Observe that the term

$$
(|X|)^{\frac{i+j}{2}-t} \Lambda\left(\lambda, l, p^{+}\right)
$$

in (26) above depends only on $i, j, t, l, s, p$ (and not on $(\mathcal{A}, f, B)$ ). It thus follows from Theorem 4.3 and Theorem 2.1 (iii) that each SSJC in $J_{X}(n, k, s, p)$ is closed under all the operators $\mathcal{M}_{i, j}^{t, l}$ and that all these SSJC's give rise to identical blocks. That the image of $\Phi$ consists of all such block diagonal matrices follows from the dimension count (5) and Lemma 4.1.

(iv) Follows from Theorem 4.3 and Theorem 2.1 (iv).

Theorem 4.4. (Ceccherini-Silberstein, Scarabotti, and Tolli [2]) Let $0 \leqslant i \leqslant n$, $(t, l) \in \mathcal{I}_{X}(n, i)$, and $(k, s, p) \in \mathcal{J}_{X}(n, i)$ with $p=\left(p_{1}, \ldots, p_{m}\right)$. Set $p_{0}=t-s$ and $p^{+}=\left(p_{0}, p_{1}, \ldots, p_{m}\right)$. The eigenvalue of $M_{i, i}^{t, l}$ on $V_{X}(n, i, k, s, p)$ is

$$
(|X|)^{i-t} \Lambda\left(\lambda, l, p^{+}\right)\left\{\sum_{u=0}^{n-s}(-1)^{u-t+s}\left(\begin{array}{c}
u \\
t-s
\end{array}\right)\left(\begin{array}{c}
n-k-u \\
i-s-u
\end{array}\right)\left(\begin{array}{c}
i-k \\
i-s-u
\end{array}\right)\right\} .
$$

Proof. Follows from substituting $j=i$ in Theorem 1.1 (iv) and noting that

$$
\left(\begin{array}{c}
n+s-2 k \\
i-k
\end{array}\right)^{-1}\left(\begin{array}{c}
n+s-2 k \\
u+s-k
\end{array}\right)\left(\begin{array}{c}
n-k-u \\
i-s-u
\end{array}\right)=\left(\begin{array}{c}
i-k \\
i-s-u
\end{array}\right) .
$$




\section{Acknowledgement}

The research of the first author was supported by the Council of Scientific and Industrial Research, Government of India.

\section{References}

[1] Bachoc, C., Passuello, A., Vallentin, F., Bounds for projective codes from semidefinite programming, Advances in Math. of Communications 7, 127-145 (2013).

[2] Ceccherini-Silberstein, T., Scarabotti, F., Tolli, F., Trees, wreath products, and finite Gelfand pairs, Adv. in Math 206, 503-537 (2006).

[3] Ceccherini-Silberstein, T., Scarabotti, F., Tolli, F., Representation theory and harmonic analysis of wreath products of finite groups, Cambridge University Press (2014).

[4] Delsarte, P., An algebraic approach to the association schemes of coding theory, Philips Res. Rep. Suppl. 10, (1973).

[5] Dunkl, C. F., A Krawtchouk polynomial addition theorem and wreath product of symmetric groups, Indiana Univ. Math. J. 26, 335-358 (1976).

[6] Gijswijt, D., Schrijver, A., Tanaka, H., New upper bounds for nonbinary codes based on the Terwilliger algebra and semidefinite programming, J. Comb. Theory, Ser. A 113, 1719-1731 (2006).

[7] Macdonald, I. G., Symmetric functions and Hall polynomials (2nd Edition), Oxford University Press (1995).

[8] Schrijver, A., New code upper bounds from the Terwilliger algebra and semidefinite programming, IEEE Tran. Information Theory 51, 2859-2866 (2005).

[9] Srinivasan, M. K., Symmetric chains, Gelfand-Tsetlin chains, and the Terwilliger algebra of the binary Hamming scheme, J. Algebraic Comb. 34, 301-322 (2011).

[10] Srinivasan, M. K., Notes on explicit block diagonalization, in Combinatorial matrix theory and generalized inverses of matrices, Springer, (2013).

[11] Srinivasan, M. K., The Goldman-Rota identity and the Grassmann scheme, Electronic J. Comb. 21(1), \#P37 (2014).

[12] Stanley, R. P., Enumerative Combinatorics - Volume 1 (Second Edition). Cambridge University Press (2012).

[13] Tarnanen, H., Aaltonen, M., Goethals, J. -M., On the nonbinary Johnson scheme, European J. Comb. 6, 279-285 (1985). 